\title{
Anti-malaria drug development targeting the M1 alanyl and M17 leucyl aminopeptidases
}

\author{
Karine Thivierge, ${ }^{\mathrm{a}}$ Rency T. Mathew, ${ }^{\mathrm{a}}$ Desire M. M. Nsangou, ${ }^{\mathrm{a}}$ Fabio Da Silva, ${ }^{\mathrm{a}}$ Sophie \\ Cotton, ${ }^{a}$ Tina S. Skinner-Adams, ${ }^{\text {b,c }}$ Katharine R. Trenholme, ${ }^{\text {b,c }}$ Christopher L. Brown, ${ }^{\text {d,e }}$ \\ Colin M. Stack, ${ }^{\mathrm{f}}$ Donald L. Gardiner ${ }^{\mathrm{b}, \mathrm{d}}$ and John P. Dalton ${ }^{\mathrm{a}} *$ \\ ${ }^{a}$ Institute of Parasitology, McDonald Campus, McGill University, 21111 Lakeshore Road, Sainte \\ Anne de Bellevue, QC H9X 3V9, Canada \\ ${ }^{b}$ Malaria Biology Laboratory. Queensland Institute of Medical Research, 300 Herston Rd, \\ Herston, QLD, Australia \\ ${ }^{c}$ School of Medicine, University of Queensland. St Lucia, 4072 QLD, Australia \\ ${ }^{d}$ School of Biomolecular and Physical Sciences. Griffith University, Nathan 4111 QLD, Australia \\ ${ }^{e}$ Eskitis Institute for Cell and Molecular Therapies, Griffith University, Nathan 411 QLD, \\ Australia \\ ${ }^{f}$ School of Biomedical and Health Sciences, University of Western Sydney, Narellan Road, \\ Campbelltown, NSW, Australia \\ E-mail: john.dalton@mcgill.ca
}

\section{Dedicated to Prof. Pawel Kafarski to honor the achievements within his career}

\begin{abstract}
The M1 alanyl aminopeptidase and M17 leucyl aminopeptidase are critical to the growth and development of malaria parasites inside host erythrocytes. Potent aminopeptidase inhibitors kill malaria parasites in culture and are also active in vivo against murine malaria. Functional recombinant enzyme studies have been used to decipher the three-dimensional structures of both enzymes that together with new and specific inhibitors are facilitating structure-activityrelationship (SAR) and functional studies. Here we review the progress made in our knowledge of these two enzymes which is bringing them closer to being validated anti-malarial drug targets.
\end{abstract}

Keywords: Aminopeptidases, anti-malarials, enzymes, malaria, peptidases, plasmodium

\section{Table of Contents}

1. Introduction

2 M1 Alanyl Aminopeptidase (PfM1AAP) and M17 Leucine Aminopeptidase (PfM17LAP) 
3. Production of Functionally-active Recombinant $P f \mathrm{M} 1 \mathrm{AAP}$ and $P f \mathrm{M} 17 \mathrm{LAP}$, and their Comparative Biochemistry

4. Are $P f \mathrm{M} 1 \mathrm{AAP}$ and $P f \mathrm{M} 17 \mathrm{LAP}$ Targets for Anti-malarial Drugs? - Proof of Concept

5. Do $P f \mathrm{M} 1 \mathrm{AAP}$ and $P f \mathrm{M} 17 \mathrm{LAP}$ Function in the Same or Different Cellular Compartment(s)?

6. Are $P f \mathrm{M} 1 \mathrm{AAP}$ and/or $P f \mathrm{M} 17 \mathrm{LAP}$ Targets for Anti-malarial Drug Development?

7. The PfM1AAP and PfM17LAP Active Site Pockets

8. Conclusion

\section{Introduction}

Malaria is endemic in about 106 countries and threatens half of the world's population. ${ }^{1-3}$ There are over 300 million cases per year and although current concerted interventions have proved successful in reducing annual deaths these still range between 850,000 and 1 million each year. ${ }^{3-5}$ Malaria is caused by protozoan parasites of the genus Plasmodium. While five species of Plasmodium infect humans, P. falciparum is responsible for most deaths, particularly in children and pregnant women in the African continent. ${ }^{3-5} P$. falciparum malaria is transmitted by infected female Anopheline mosquitoes that inject infective sporozoites into the human bloodstream when they bite. These sporozoites migrate to the liver where they invade hepatocytes and form exoerythrocytic schizonts, each containing approximately 30,000 merozoites. After a period of between 8 and 11 days, the schizont-infected hepatocytes rupture releasing mature merozoites into the bloodstream where they infect host erythrocytes. Within the erythrocytes the parasites progress through strictly regulated phases of asexual development known as rings (22-24 hours), trophozoites (10-12 hours) and schizonts (6-10 hours). Mature schizonts develop into as many as 32 merozoites that, following rupture of the erythrocyte, are released and quickly invade other erythrocytes to begin the next cycle. This repeated cycle leads to a rapidly increasing number of infected cells (parasitaemia) which, if uncontrolled, can lead to death. ${ }^{3}$

The clinical manifestations of malaria occur when parasites rupture from the erythrocytes. It is not surprising, therefore, that many new anti-malarial drug development strategies focus on targeting the asexual intra-erythrocytic stages of Plasmodium development. ${ }^{2,3}$ Intraerythrocytic parasites are metabolically active and breakdown $65-75 \%$ of host cell haemoglobin in a hydrolytic process that ultimately releases free aminoacids that are used by the parasite in protein synthesis. ${ }^{6,7}$ Haemoglobin digestion is also considered essential for the maintenance of osmotic pressure within the infected erythrocyte, a process that is believed to prevent premature erythrocyte lysis (colloid osmotic hypothesis). ${ }^{7}$ However, as the inhibition of haemoglobin digestion with peptidase inhibitors does not cause premature osmotic lysis of infected cells, Naughton $e t a l .{ }^{8}$ suggested that its primary function is nutritional. Interestingly, Krugliak et al. ${ }^{9}$ found that only $16 \%$ of degraded haemoglobin is used by parasites for protein synthesis, the rest being converted into haemozoin or disposed of by alternative mechanisms. Malaria parasites also have the ability to synthesize several aminoacids de novo (including asparagine, glutamine, 
glycine, proline, aspartate and glutamate) and can acquire additional aminoacids from exogenous sources such as host plasma. ${ }^{10,11}$ Isoleucine, which is the only aminoacid absent from human haemoglobin, is obtained from outside of the red blood cell by exchanging it with leucine, an abundant aminoacid in haemoglobin ${ }^{12}$. Malaria parasites have been shown to grow in culture medium lacking all aminoacids except isoleucine. ${ }^{11,13}$

Inside the erythrocyte the parasite resides within its own membrane-protected space, known as the parasitophorus vacuole (PV), and is therefore not in direct contact with the red cell milieu. ${ }^{6,7,13,14}$ The contents of the host erythrocyte cytoplasm are actively taken into the parasite via cytostomes and then transported in vesicles to a specialized organelle known as the food or digestive vacuole (DV) for breakdown. ${ }^{6,13,14}$ The DV is maintained at a low pH, estimated at 5.2, at which most of the DV proteases function best, although a low $\mathrm{pH}$ is also likely to induce structural relaxation or denaturation of haemoglobin protein making it more susceptible to proteolytic hydrolysis. ${ }^{6,13,14}$ By contrast, the $\mathrm{pH}$ of the malaria cell cytoplasm is $7.2 .^{14-16}$

Catabolism of host haemoglobin in the DV involves a number of endopeptidases of various mechanistic classes; these include several aspartic proteases termed plasmepsins (plasmepsins I, II, IV and histo-aspartic protease), three cysteine proteases termed falcipains (falcipains 2, 2' and 3 ) and a single metallo-protease termed falcilysin. The combined and simultaneous action of these proteases degrades haemoglobin into small peptides of about 8-10 aminoacids long. ${ }^{17-20}$ As the DV falcipains and plasmepsins have historically been a focus of anti-malaria drug discovery efforts much is known about their biochemistry, structure and function. ${ }^{13,18,19}$ However, knockout experiments have shown that the DV plasmepsins and falcipains 2 and 2 ' are individually not essential to parasite survival, and because of this redundancy between the function of these enzyme classes it has proved difficult to develop drugs that effectively block the digestive process within the DV. ${ }^{21-24}$

Peptides produced in the DV may be further processed to dipeptides by a dipeptidyl aminopeptidase I, an orthologue of mammalian cathepsin C. ${ }^{25,26}$ Small peptides and dipeptides may also be reduced to free aminoacids within the DV and/or transported to the parasite cytosol for processing by aminopeptidases. ${ }^{20,27-33}$

Aminopeptidases are essential in releasing aminoacids from haemoglobin-derived peptides and are thus central to the growth and development of malaria parasites in the erythrocyte. ${ }^{31,34}$ The $P$. falciparum genome encodes five aminopeptidases that are potential candidate enzymes for releasing free aminoacids from haemoglobin-derived peptides, ${ }^{34}$ these include three highly specific enzymes, a post-prolyl aminopeptidase (PfAPP) that is located in the DV and an aspartic aminopeptidase (M18AAP) and a prolyl aminopeptidase (S33PAP) located in the cytoplasm. These three enzymes may function in concert with the M1 alanyl aminopeptidase (M1AAP) and an M17 leucine aminopeptidase (M17LAP) that are the subject of this review. 


\section{M1 Alanyl Aminopeptidase (PfM1AAP) and M17 Leucine Aminopeptidase (PfM17LAP)}

The presence of aminopeptidase activity in malaria cell extracts with a $\mathrm{pH}$ optimum in the neutral range was first described over thirty years ago, ${ }^{35-39}$ and was postulated to be involved in the release of free aminoacids from host hemoglobin by Slomianny et al. ${ }^{37}$ The proteins responsible for the neutral aminopeptidase activity in extracts obtained from various malaria species have been characterized as ranging from 63 to $180 \mathrm{kDa}^{27,29,35-39}$ Biochemical analysis of soluble extracts from $P$. falciparum by Gavigan et al. ${ }^{29}$ identified high levels of neutral aminopeptidase activity that could release aminoacids from synthetic peptides representative of the products of haemoglobin digestion in the DV. This activity had a clear preference for synthetic substrates containing N-terminal leucine or alanine residues which was relevant since these are the most abundant residues in human haemoglobin $(\sim 24 \%) .^{27-29}$

The data from most studies implied that aminopeptidase activity is associated with the parasite cytoplasm, although ultrastructural cytochemical studies by Slomianny et al. ${ }^{37}$ indicated aminopeptidase activity may be present within the DV. We suggested that the biochemical characteristics of malaria aminopeptidases in cell extracts were consistent with an enzymatic function within the parasite cytosol and not in the low $\mathrm{pH}$ environment of the DV. ${ }^{27-29}$ Neither Gavigan et al. ${ }^{29}$ nor Kolakovich et al. ${ }^{20}$ detected aminopeptidase activity in the DV and thus it was proposed that haemoglobin-derived peptides generated within the DV must be transported to the cytosol for processing to free aminoacids by the neutral aminopeptidases. This proposal has been questioned recently by Ragheb et $a l .^{33}$ (see below).

Prior to completion of the $P$. falciparum genome sequencing project the exact number and type of aminopeptidases in this malaria parasite were unknown. An active component partially purified from malaria extracts by Gavigan et $a .^{29}$ using size and bestatin affinity chromatography had a substrate specificity in keeping with a leucyl aminopeptidase. Florent et $a l .^{40}$ also described a similar type of activity and were first to isolate and characterize a gene that encoded a malaria aminopeptidase which was identified as a member of the M1 family of alanyl aminopeptidases. Publication of the $P$. falciparum $26 \mathrm{Mb}$ genome allowed the identification of an additional enzyme potentially responsible for the neutral aminopeptidase activity in malaria extracts. ${ }^{40}$ As it turned out, the parasite possessed two single-copy genes encoding metalloexopeptidases with similar activities, although their structure and classification are quite different. The first, is a $122 \mathrm{kDa}$ M1-family alanyl aminopeptidase (PfM1AAP) with a transmembrane portion in its N-terminal region (as originally described by Florent et $a l .{ }^{40}$ ), and the second is a $67.8 \mathrm{kDa}$ M17-family leucyl aminopeptidase (PfM17LAP) with no trans-membrane region. ${ }^{31}$ 


\section{Production of Functionally-active Recombinant PfM1AAP and PfM17LAP, and their Comparative Biochemistry}

Functionally active recombinant PfM1AAP (rPfM1AAP) and M17LAP (rPfM17LAP) have been produced and have proven of critical importance to the detailed characterization of the physicobiochemical properties and three-dimensional structure of these enzymes. ${ }^{41-45}$ Recombinant expression in E. coli and/or insect cells was achieved by optimizing the gene codon usage, deleting regions with low complexity (including the trans-membrane portion in the case of PfM1APP) and removal of potential N-glycosylation sites. ${ }^{41-43}$ Consistent with their mammalian homologues, rPfM1AAP was shown to be monomeric whereas rPfM17LAP is a homo-hexamer of approximately $320 \mathrm{kDa} .{ }^{42,43}$

Comparative biochemical studies have been performed with soluble malaria extracts and recombinant enzyme. These studies demonstrated that (a) the recombinant enzymes exhibit similar pH optima, $\mathrm{pH}$ stability and kinetic parameters against peptide substrates to their corresponding native forms, and (b) both native and recombinant enzymes have similar inhibition constants for the aminopeptidase inhibitors (such as bestatin, $\mathbf{1}$ and hPheP $\left[\mathrm{CH}_{2}\right] \mathrm{Phe}, \mathbf{3}$, Figure 1, see below). Since rPfM1AAP and rPfM17LAP had similar properties to their native enzymes they were suitable for structural characterization and use in substrate preference kinetics/inhibitor studies. $^{41-43}$

Analysis of enzyme kinetic data revealed that PfM1AAP and PfM17LAP have overlapping substrate specificities. ${ }^{42,43}$ PfM1AAP substrate specificity is broader than PfM17LAP's. PfM1AAP can rapidly hydrolyse N-terminal leucine, alanine, arginine, and lysine; it can also cleave phenylalanine, tyrosine, serine, and asparagine at lower catalytic rates. In contrast, PfM17LAP exhibits an almost exclusive specificity for leucine; hydrolysis of N-terminal alanine, proline or phenylalanine is poor, and the enzyme is unable to cleave N-terminal valine, glycine, asparagine, glutamine, isoleucine or arginine. Because the aminoacid arginine is readily and uniquely cleaved by PfM1AAP, an assay for the specific detection of this enzyme in malaria extracts using the fluorogenic peptide substrate arginine-4-aminomethylcourmarin was developed. $^{31,42}$

Curley et $a .^{27}$ showed that neutral aminopeptidase activity in malaria cell extracts is dependent on metal ions. While both PfM1AAP and PfM17LAP possess metal ions in their active sites (presumably zinc by analogy with their mammalian counterparts), PfM1AAP binds a single metal ion whereas PfM17LAP binds two ions. Moreover, metal chelation and binding studies have demonstrated that one of the two active site metal ions in PfM17LAP is readily exchanged with other metals (such as $\mathrm{Co}, \mathrm{Mg}$ and $\mathrm{Mn}$ ) or lost by dialysis with EDTA (site 1) whereas the second is more tightly bound (site 2). ${ }^{46}$ The single metal binding site of PfM1AAP exhibits tight-binding properties. These observed differences in metal ion binding properties are important for the interpretation of the mode and mechanism of action of aminopeptidase inhibitors since these commonly contain a 'warhead' that recognizes the active site of the enzyme and a specific functional ligand that complexes with the metal ion(s). ${ }^{47}$ In addition, the 
difference in the susceptibility of the two enzymes to metal ion concentration may be important in the regulation of their activities in malaria parasites. ${ }^{31,46}$

\section{Are PfM1AAP and PfM17LAP Aminopeptidases Targets for Anti-malarial Drugs? Proof-of-Concept}

Early studies by Nankya-Kitaka et $a l^{28}$ and Gavigan et $a .^{29}$ demonstrated that the aminopeptidase inhibitor bestatin (1), a natural Phe-Leu dipeptide analog derived from the fungus Streptomyces olivoretticuli, and its derivative nitrobestatin (2, Figure 1) were potent inhibitors of neutral aminopeptidase activity in malaria cell extracts and could kill parasites (including chloroquine-resistant strains) in in vitro assays. These compounds are primarily active against the intraerythrocytic trophozoite stage, which exhibit the highest levels of neutral aminopeptidase activity. Using the recombinant enzymes we showed that bestatin and nitrobestatin were potent inhibitors of both $P$. falciparum PfM1AAP and PfM17LAP. ${ }^{31,41-43}$ Recently, Harbut et $a l .^{45}$ used bestatin-based activity probes to demonstrate that PfM1AAP and PfM17LAP are the only likely targets for bestatin in malaria parasites.

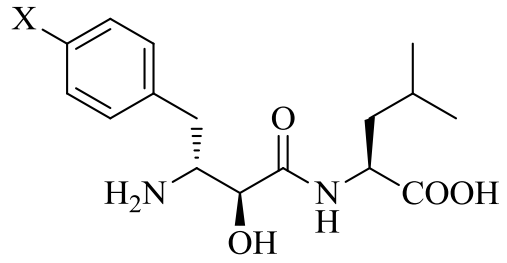

1, $\mathrm{X}=\mathrm{H}$, bestatin

$\mathbf{2}, \mathrm{X}=\mathrm{NO}_{2}$, nitrobestatin<smiles>O=C(NO)/C(=C\c1ccccc1)C(=O)NCc1ccc(F)cc1</smiles>

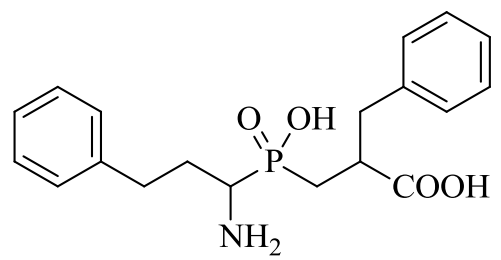

3, hPhe $\left[\mathrm{CH}_{2}\right] \mathrm{Phe}$

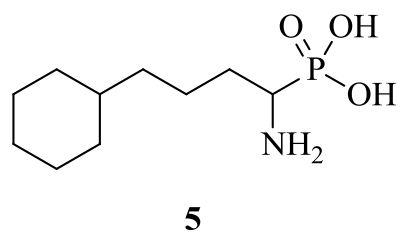

Figure 1. A selection of aminopeptidase inhibitors investigated for their antimalarial activity.

Using computer-aided design Grembecka et $a l .^{48}$ developed a series of phosphinate dipeptide analogs for the purpose of selectively targeting mammalian aminopeptidase N. These were also shown to be potent inhibitors of PfM1AAP and PfM17LAP. One of these inhibitors hPheP[ $\left[\mathrm{CH}_{2}\right]$ Phe (3) inhibited recombinant aminopeptidases more effectively than bestatin and was also effective in vitro against $P$. falciparum. ${ }^{31,49}$ Another compound hPheP $\left[\mathrm{CH}_{2}\right]$ Gly did not 
inhibit either enzyme and had no activity against parasites in culture demonstrating a correlation between enzyme inhibition and parasite killing.

Flipo et $a .^{50}$ developed a number of dual function amide-hydroximate compounds that consisted of an $\alpha$-substituent, a hydroxamic acid terminus (that complexes with the active site $\mathrm{Zn}$ ion), and an amide that was structurally diversified to produce potent inhibitors of PfM1AAP (exemplified by structure 4, Figure 1). While many of these novel compounds had $\mathrm{IC}_{50}$ values against native PfM1AAP (isolated from cell extracts) in the low nanomolar range, two exhibited an impressive (>100-fold) specificity against the malaria enzyme compared to the mammalian homolog (porcine kidney APN) and killed $P$. falciparum malaria parasites in culture $\left(\mathrm{IC}_{50}=13-\right.$ $59 \mu \mathrm{M})$. The activity of these compounds against the PfM17LAP was not determined.

Cunningham et al. ${ }^{51}$ reported the evaluation of a series of $\alpha$-aminoalkylphosphonate and phosphonopeptide inhibitors, designed against mammalian M17LAP, for their anti-malaria activity. They then correlated the $\mathrm{IC}_{50} \mathrm{~s}$ of these compounds with their inhibitory activity against aminopeptidase activity in malaria cell extracts, and against recombinant PfM17LAP. A linear relationship between aminopeptidase inhibition and parasite killing was observed (best compound 5, Figure 1, $\mathrm{IC}_{50}=14 \mu \mathrm{M}$ ), although all compounds appeared to inhibit both $P f$ M1AAP and PfM17LAP and none exhibited superior activity compared to bestatin.

Skinner-Adams et al. ${ }^{49}$ performed in vivo studies with $\mathbf{1}$ and $\mathbf{3}$ (see above) using a $P$. $c$. chabaudi murine malaria model. Intraperitoneal administration of phosphinate $\mathbf{3}$ was shown to delay the appearance of parasites by 3 days and reduced parasitaemia by $96.8 \%$ compared to PBS-administered controls with a complete clearance of infection in the subsequent four days. Compound 3 was more effective than treatment with bestatin, which reduced peak parasitaemia by $34 \%$. These treatments did not cause any apparent side effects in the mice. In the same study, the anti-malarial chloroquine delayed the appearance of parasites by six days, and reduced median peak parasitaemia by $93 \%$ on day 14 with a complete clearance of parasites over the following four days. The results of these in vivo studies, albeit performed in mouse models, are significant in that they provided the first and vital proof-of-concept for considering the $P f$ M1AAP and PfM17LAP aminopeptidases as targets at which a new class of anti-malarial drug could be developed.

\section{Do $P f M 1 A A P$ and $P f M 17 L A P$ Function in the Same or Different Cellular Compartment(s)?}

Determining the location of the two neutral aminopeptidases within the malaria parasite is important in understanding their function, and is also relevant for the design of anti-malaria drugs that must first pass through several membranes (erythrocyte, parasitophorous and parasite surface) before entering the parasite to reach their target enzyme(s). PfM17LAP was detected as a $68-70 \mathrm{kDa}$ protein in soluble extracts of $P$. falciparum malaria parasites, immunocytochemical studies located the enzyme to the parasite cytoplasm. ${ }^{41,52}$ This location has been confirmed by 
confocal microscopy of live transfected parasites expressing fluorescently-tagged $P f M 17 L A P^{30,41,52}$. Using specific inhibitors of PfM17LAP, Harbut et al ${ }^{45}$ showed that killing of $P$. falciparum in culture occurs early in the intraerythrocytic cycle (ring stages) prior to the onset of significant haemoglobin digestion suggesting that this enzyme does not play a part in this process. Within the parasite cytosol, PfM17LAP may be important in the terminal stages of protein/peptide turnover. ${ }^{30,31,41,45}$ The almost exclusive specificity of PfM17LAP for leucine suggests that this enzyme may be essential for the generation of free leucine that can be exchanged for extracellular isoleucine ${ }^{12,31}$, an aminoacid that cannot be synthesized by the parasite and is the only aminoacid not found in human haemoglobin. Naughton et al. ${ }^{8}$ reported that bestatin, at 5- and 25 -fold its $\mathrm{IC}_{50}$, reduced the up-take of isoleucine into $P$. falciparum cells in vitro by $20-25$ and $50 \%$, respectively, which may support this idea.

Allary et al. ${ }^{53}$ showed that $P f \mathrm{M} 1 \mathrm{AAP}$, which contains a transmembrane domain, is expressed in parasite extracts as a $120 \mathrm{kDa}$ protein. This protein is proteolytically processed at the $\mathrm{N}$ terminus into a fragment of $96 \mathrm{kDa}$ lacking the trans-membrane domain and also a $68 \mathrm{kDa}$ fragment that is cleaved at both $\mathrm{N}$ - and C-terminal ends. Anti-peptide antibodies that react with all three forms localized the PfM1AAP within the cytosol; no localization was observed within the DV although distinct accumulation around the DV was observed. A more recent study from this laboratory described how the $96 \mathrm{kDa} P f \mathrm{M} 1 \mathrm{AAP}$ is trafficked outside the parasite into the PV space before being converted to the $68 \mathrm{kDa}$ form and re-directed into the parasite cytoplasm and marginally to the DV (cell fractionation and immunoblotting experiments estimated that about $16 \%$ of the $68 \mathrm{kDa}$ form ends up in the DV). ${ }^{54}$ Immunocytochemistry studies in our laboratory using anti-peptide serum and live parasites transfected with GFP-tagged constructs indicated that PfM1AAP was located in the parasite cytosol. ${ }^{42}$ Together with its observed $\mathrm{pH}$ optimum for activity at neutral $\mathrm{pH}$ we interpreted these findings as indicative of a cytoplasmic function for $P f$ M1AAP, possibly alone and/or in concert with $P f \mathrm{M}_{17} \mathrm{LAP}^{31,41}$ and another cytoplasmic aminopeptidase $P f$ M18AAP that exhibit as highly specific specificity for glutamate and aspartate. $^{55}$

By contrast, studies by Dalal and Klemba ${ }^{30}$ using $P$. falciparum parasites stably expressing yellow fluorescent protein-tagged $P f$ M1AAP localized this enzyme to the DV and nucleus only. These cellular locations were further supported by immunoelectron microscopy ${ }^{33}$, and therefore Klemba and colleagues proposed that the PfM1AAP functions within the DV, releasing aminoacids from peptides generated by other endopeptidases (falcipain, plasmepsins and dipeptidase), and may have a separate role within the nucleus. In support of this theory, Ragheb et al $^{33}$ showed that while PfM1AAP exhibited less activity in the $\mathrm{pH}$ range $5.0-5.5$ than at a neutral $\mathrm{pH}$ environment (consistent with previously published data ${ }^{42}$ ), it is still nevertheless stable and functional at acidic $\mathrm{pH}$. The turnover rate $\left(k_{\mathrm{cat}}\right)$ of the enzyme did not alter dramatically at $\mathrm{pH} 5.5$, and although the $K_{m}$ did increase with decreasing $\mathrm{pH}$ the authors suggested that this could be compensated for by an increased substrate concentration within the lumen of the DV. Further support for the suggested function of PfM1AAP within the DV was provided by Harbut et al. ${ }^{45}$ who observed swelling of this organelle and inhibition of proteolysis 
of haemoglobin-derived peptides when parasites where exposed to a PfM1AAP-specific bestatinlike inhibitor in culture.

Earlier studies by Gavigan et al. ${ }^{29}$ using electron microscopy did not show any effect on the DV when parasites where incubated in vitro with bestatin. Ragheb et al. ${ }^{33}$ attributed differences between their immunocytochemical localization observations (DV and nucleus) and that of Allary et al. $^{53}$ (cytoplasm) to the use of different fixation methods. In an attempt to equate the collective immunocytochemical and biochemical data we have suggested that PfM1AAP could be associated with the DV by insertion within the DV membrane with the catalytic domain orientated towards the cytoplasm; in this location the enzyme may readily cleave peptides emerging from the DV at its optimum $\mathrm{pH}$ for activity, ${ }^{31}$ and inhibition of its function could conceivably perturb the DV function and structure. A recent proteomics study by Lamarque $e t$ al. ${ }^{56}$ detected PfM1AAP in DV-enriched (12-fold) preparations which seemingly provided support for its location in this organelle, however, these preparations also contained the cytoplasmic PfM17LAP. In conclusion, several issues regarding the cellular location and, hence, function of PfM1AAP need further elucidation.

\section{Are PfM1AAP and PfM17LAP Targets for Anti-malarial Drug Development?}

PfM1AAP and PfM17LAP are each encoded by single copy gene. Both aminopeptidase genes are refractory to genetic deletion strongly suggesting that knocking-out either gene is lethal to the parasite, and that both are required for parasite survival in the erythrocyte. ${ }^{30,34} \mathrm{We}$ also generated transgenic $P$. falciparum parasites that over-expressed functionally active aminopeptidases in the cytoplasm and showed that these parasites were less susceptible to killing by bestatin (1) and hPheP[CH $\left.\mathrm{CH}_{2}\right]$ Phe (3) when compared to wild-type parasites. ${ }^{42,52}$ These data imply that both $P f$ M1AAP and PfM17LAP are targets for the anti-malarial activity of these two broad-spectrum aminopeptidase inhibitors.

Since most inhibitors used in the study of the malaria aminopeptidases block the activity of both PfM1AAP and PfM17LAP it remained unclear from reported in vitro and in vivo tests whether one or both enzymes was the targets of the aminopeptidase inhibitor-mediated killing of malaria parasites. However, Harbut et $a l .{ }^{45}$ recently identified a series of activity-based probes (ABP) based on the scaffold of bestatin that preferentially (12-15 fold) inhibited either PfM1AAP or PfM17LAP (see next section). By employing these compounds in in vitro studies they demonstrated that (a) inhibition of either enzyme results in parasite death, supporting the idea that both enzymes are potential anti-malaria targets, (b) inhibition of PfM1AAP caused DV swelling in latter stages of asexual development whereas (c) inhibition of PfM17LAP halted the development of ring stage parasites before the main process of haemoglobin degradation takes place. These observations indicate that PfM17LAP and PfM1AAP perform different functions 
within the malaria parasite and that each could be individually targeted for drug development (see previous Section).

\section{The PfM1AAP and PfM17LAP Active Site Pockets}

The structures of PfM1AAP and PfM17LAP are central to understanding both the mechanism of action of currently available inhibitors and to the future development of anti-malaria drugs targeting these enzymes. Using our recombinant proteins (see above) McGowan et al. ${ }^{42,43}$ reported the X-ray crystal structures of the monomeric PfM1AAP (lacking the first 194 residues which contains the trans-membrane domain $)^{42}$ and the hexameric leucine PfM17LAP, ${ }^{43}$ alone and in complex with bestatin and the phosphinate analogue hPheP $\left[\mathrm{CH}_{2}\right] \mathrm{Phe}$. More recently, the same recombinant proteins were employed in the SAR studies of novel bestatin-based inhibitor libraries and activity-based probes by Valmourougane et al. ${ }^{44}$ and Harbut et al. ${ }^{45}$

The PfM1AAP consists of four domains; the highly-conserved zinc-binding $\mathrm{H}^{496} \mathrm{EYFHX}_{17} \mathrm{KE}^{519}$ motif and the substrate recognition $\mathrm{G}^{460}$ AMEN motif are contained within domain IV, or the catalytic domain (Figure 2). These zinc binding and substrate coordinating motifs lie within the buried active site pocket that has access to the external milieu via two openings. One of these is formed by a helical superstructure within domain IV that forms a $28 \AA$ channel that is large enough to permit peptide substrate entry to the active site. The second is a shallow $8 \AA$ long channel formed by the juncture of domains I and IV and is likely to be the exit point for released aminoacids. ${ }^{42}$

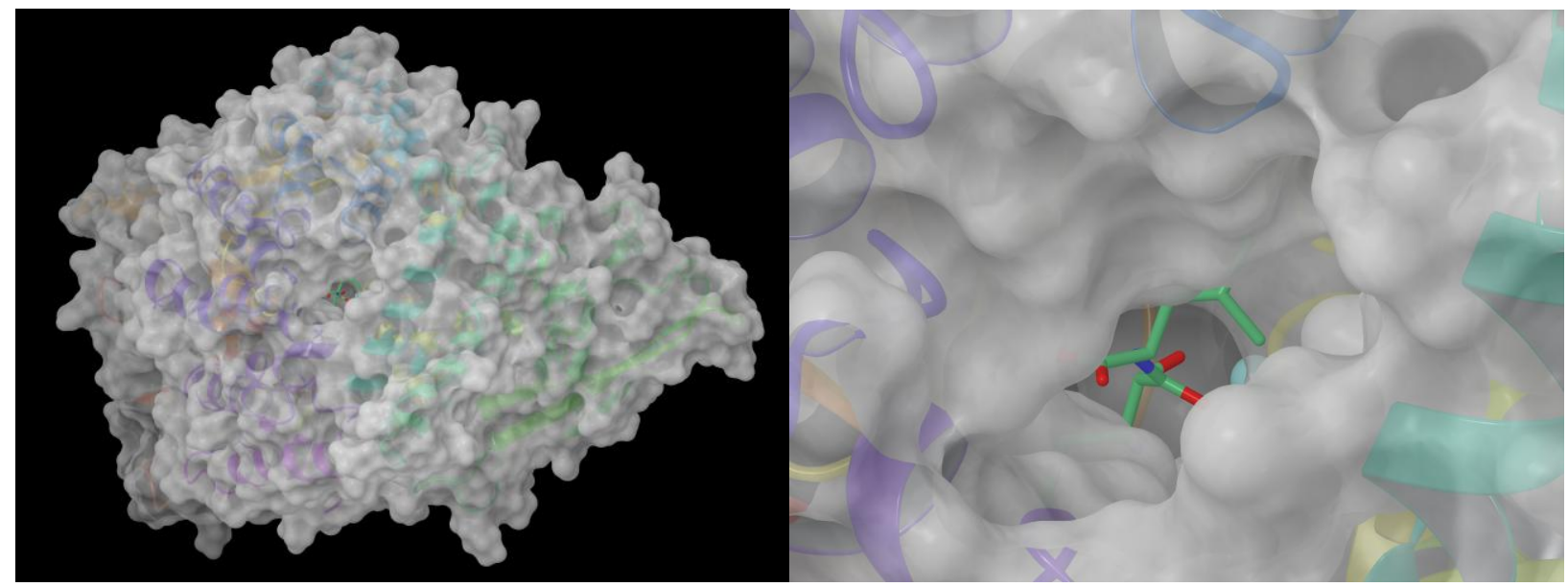

Figure 2. Molecular surface representation of rPfM1AAP (from pdb code 3EBH) bound to bestatin (left). The close-up view of the large C-terminal channel illustrates the location of the inhibitor within the binding site of the protein (right). 
Molecular dynamic simulation studies by Jones et al. ${ }^{57}$ of an apo-PfM1AAP and a ligandbound complex state revealed that substrate binding in the catalytic domain are coupled to global conformational changes in the protein. A C-terminus $\alpha$-helix 10 and downstream loop proximal to the opening of the C-terminal channel undergoes the largest fluctuations together with the opening of the shallow $8 \AA$ long channel N-terminal small channel. It was suggested that the function of the $\alpha$-helix 10 "arm" is to move peptides into the cavity as free aminoacids are released at the $\mathrm{N}$-terminal small opening. Thus, a concerted conformational dynamic within the catalytic domain II ensures that as the N-terminal small channel opens to release product the $\alpha$ helix 10 facilitates the movement of the peptide ligand one aminoacid at a time into the cavity by acting in a 'ratchet-like' fashion. The internal chamber may act as a vestibule or 'waiting room' for smaller peptides that get shunted along toward the active site like an assembly line. ${ }^{57}$

The S1 active site pocket of PfM1AAP is lined principally by hydrophobic residues $\left(\mathrm{Gln}^{317}\right.$, $\mathrm{Val}^{459}$, $\mathrm{Tyr}^{575}$ and $\mathrm{Met}^{1034}$ ), while a polar residue $\mathrm{Glu}^{572}$ located at the base of the S1 pocket is available to interact with the P1 side groups of the substrate. ${ }^{42}$ The S1 pocket accepts hydrophobic residues while the S1' pocket, consisting of $\mathrm{Thr}^{492}, \mathrm{Val}^{493}$ and $\mathrm{Val}^{523}$, favors small aliphatic residues. Interestingly, Valmourougane et al. ${ }^{44}$ discovered that the $\mathrm{S} 1$ pocket loop, residues 570-575, is mobile and can move to accommodate larger side chains compared to its mammalian counterparts, a property that could possibility be exploited for specific inhibitor design.

PfM17LAP (monomer) possesses an N-terminal regulatory domain and a C-terminal catalytic domain that houses the active site. The two active site $\mathrm{Zn}$ ions are almost $3 \AA$ apart and are coordinated by $\mathrm{Asp}^{379}, \mathrm{Asp}^{459}$, and Glu ${ }^{461}$ (loosely bound site 1) and Lys ${ }^{374}$, Asp ${ }^{379}$, $\mathrm{Asp}^{399}$ and Glu ${ }^{461}$ (tightly bound site 2). The active sites of PfM17LAP (hexamer) are arranged in a disc-like fashion so that they are orientated inwards to form a central large catalytic cavity (Figure 3). ${ }^{43}$ In contrast to the broad substrate specificity exhibited by PfM1AAP (Leu, Ala, Phe, Arg, Val, Met), the structural data revealed that a very narrow and hydrophobic pocket formed in this enzyme accounts for its highly restricted substrate specificity (Leu and Ala). In addition, the active site of PfM17LAP does not contain any residue capable of forming hydrogen bonds with polar P1 substrate residues. Access channels formed by the $\mathrm{N}$-terminal domains of the hexamer may play a role in determining the specificity of substrates that enter the catalytic cavity and flexible loops that sit at each of the six entrances of the active sites may function to regulate substrate access. ${ }^{43}$ 


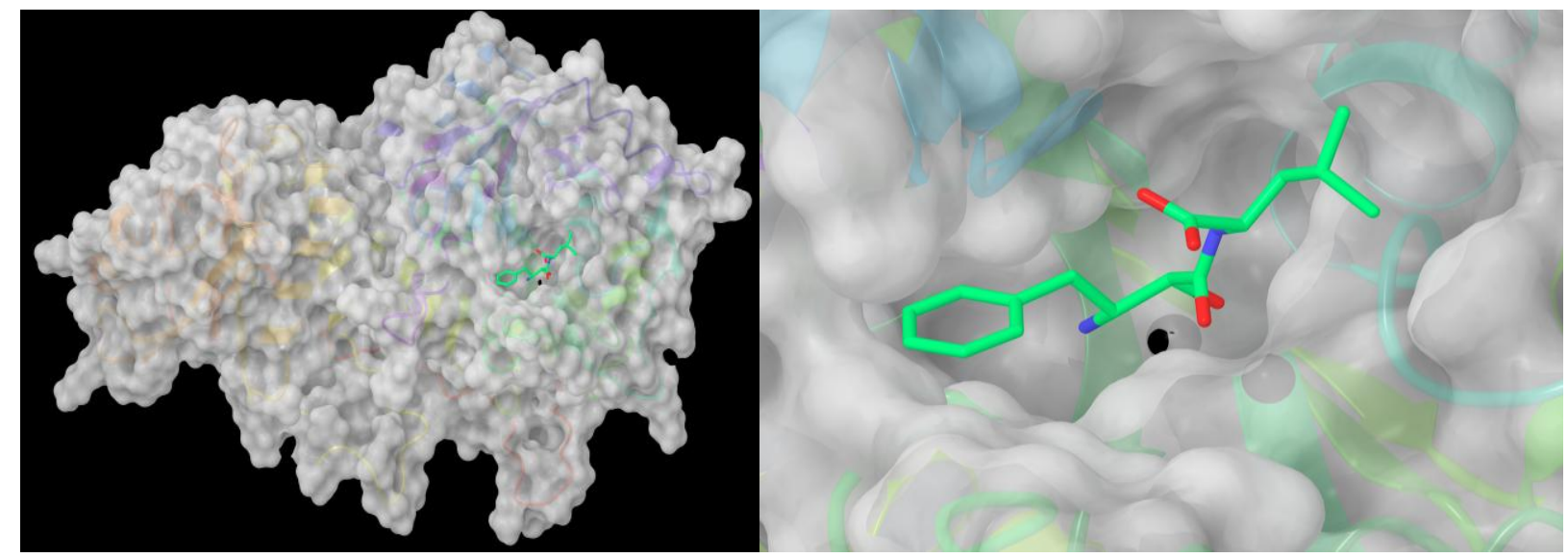

Figure 3. Molecular surface representation of rPfM17LAP monomer (from pdb code 3KR4) bound to bestatin (left). The close-up view illustrates how the inhibitor sits into the narrow active site (right).

Bestatin 1 is a slow, tight-binding reversible inhibitor of malaria aminopeptidases ${ }^{49,58}$ that contains a hydroxyl group, a central nitrogen and a carbonyl group which mediates the interaction with the active site metal ions. This inhibitor is more effective (19-fold) against PfM17LAP compared to PfM1AAP ${ }^{31}$ because it chelates two metal ions rather than one, and makes a greater number of hydrogen bonds within the active site of PfM17LAP (12 as opposed to 8 ). The synthetic phosphinate dipeptide analogue 3 which exhibits fast, tight binding characteristics, is a more potent inhibitor of PfM17LAP and PfM1AAP than bestatin and also shows a greater inhibition (6-fold) for PfM17LAP compared to PfM1AAP. ${ }^{31}$ The Phe-rings of this compound bind more favorably to both enzymes and the central phosphate group provides a hexavalent coordination of the active site metal ions ${ }^{43}$. Interestingly, the binding of $\mathrm{hPheP}\left[\mathrm{CH}_{2}\right]$ Gly to both enzymes is dramatically reduced highlighting the need for a hydrophobic residue in the $\mathrm{P} 1$ ' position. Consequently, this compound exhibits no killing against $P$. falciparum parasites in vitro. ${ }^{31,49}$

The X-ray co-crystal structure of PfM1AAP bound to the dipeptide activity-based probe (Benzyl)Tyr-Ala (termed BTA) which was designed using the bestatin scaffold and exhibited a 15-fold specificity for PfM1AAP over PfM17LAP was solved by Harbut et al. ${ }^{45}$ The structural data demonstrated that the probe binds to the aminopeptidase via a manner similar to bestatin, with only slight movement within the S1 pocket observed. Molecular modeling that employed PfM17LAP structure bound to bestatin showed that reduced inhibition of the BTA probe to PfM17LAP could be explained by a clash between the (Benzyl)Tyr residue with the narrow S1 pocket of this enzyme. Another probe, Phe-Naphthyl (termed PNAP) exhibited a 11-fold specificity for PfM17LAP over PfM1AAP and was shown by crystallography to fit neatly into the narrow active site of PfM17LAP by formed hydrophobic interactions with the S1 and S1' pockets, with some flipping of Ser550 to accommodate a $\mathrm{P}^{\prime}$ naphthyl group. The reduced 
binding of PNAP to PfM1AAP was accounted for by a clash of the P1' naphthyl group with the $\mathrm{S} 1$ ' pocket of this aminopeptidase.

\section{Conclusion}

The prevention and treatment of $P$. falciparum malaria is becoming difficult due to the spread of drug-resistant parasites. ${ }^{59}$ While a promising vaccine for malaria is currently in phase III trials, its efficacy appears to be in the order of $50 \%,{ }^{60}$ and thus malaria drugs are still critical to control and elimination programs. Artemisinin (ART) derivatives in combination with one or more longer acting antimalarial $\operatorname{drug}(\mathrm{s})$ of a distinct chemical class, for example lumefantrine, mefloquine or amodiaquine ${ }^{2-5}$ (referred to as ART-based combination therapies, ACTs) are recommended for the treatment of uncomplicated $P$. falciparum malaria in most malaria-endemic areas and are arguably our last line of defense against this devastating disease. However, strains of $P$. falciparum with reduced sensitivity to ACTs are emerging, especially in the ThaiCambodian border and in Bangladesh. ${ }^{5,61,62}$ This is of great concern and highlights the urgent need for next generation antimalarial agents that target different parasite biochemical pathways.

While the multistep process of haemoglobin digestion has been a notable target of antimalarial drug development over the last few decades, functional redundancy among enzymes with a similar mechanistic class (falcipains or plasmepsins) and between these classes has reduced the enthusiasm for the development of effective antimalarial agents targeting this system. $^{13,18,19,22}$ However, unlike the plasmepsins and falcipains, PfM1AAP and PfM17LAP are encoded by single copy genes in both human and rodent malaria species and each are critical to malaria survival and are therefore a more tractable target for antimalarial drug development. ${ }^{31,34}$ Substrate, inhibitor and metal binding studies together with immunocytochemical studies all point towards quite distinct functions for each of these aminopeptidases and offer the potential of developing combination therapies that would reduce the prospect of resistant malaria parasites arising. Furthermore, Gavigan et $a l^{63}$ showed synergistic activity between aminopeptidase inhibitors and inhibitors of the falcipains and plasmepsins (albeit much lower than that observed between inhibitors of each these two enzyme classes) indicating that combination drugs therapies with these endopeptidases could also be possible.

In addition to the PfM1AAP and the PfM17LAP, the genome of $P$. falciparum also contains other aminopeptidases with potential for anti-malaria drug development. For example, a cytoplasmic aspartyl aminopeptidase (PfM18AAP), that is the only malaria aminopeptidase capable of cleaving aspartic and glutamic acids from the $\mathrm{N}$-termini of peptides, is also present in single copy and is essential for parasite viability. ${ }^{55}$ Also in consideration are the post-prolyl aminopeptidase (PfAPP) ${ }^{32}$ and dipeptidyl peptidase (cathepsin C) ${ }^{25,26}$ that are located in the DV and central to the later stages of hemoglobin degradation.

A major challenge will be to develop compounds that exhibit selectivity for the malaria enzyme without off-target effects against their human homologs or other enzymes. It is relevant 
to point out, however, that inhibitors of mammalian M1AAP and M17LAP enzymes, including bestatin and the hydroxamic acid metallopeptidase inhibitor tosedostat, with anti-proliferative effects against certain tumor cells and are well tolerated in patients. ${ }^{64-66}$ High resolution structures of PfM1AAP and the PfM17LAP in complex possess various inhibitors has revealed the constellation of ligand-enzyme interactions and mechanism of binding that, alongside the availability of functionally active recombinant enzymes, is facilitating more detailed SAR studies and the development of new specific anti-malaria aminopeptidase inhibitors.

\section{Acknowledgements}

We would like to thank Prof. Paweł Kafarski, Dr. Artur Mucha, Dr. Jolanta Grembecka and Dr. Marcin Drag, Wrocław University of Technology, Poland, for their collaborative exchanges over the last six years. JPD is a CIHR Canada Research Chair in Infectious Diseases.

\section{References}

1. Yamey, G. Brit. Med. J. 2004, 328, 1086.

2. Schlitzer, M. Chem. Med. Chem. 2007, 2, 944.

3. World Health Organisation. World malaria report. 2010, http://www.who.int/malaria/world_malaria_report_2010/en/index.html

4. Enserink, M. Science 2008, 321, 1620.

5. White, N. J.; McGready, R. M.; Nosten, F. H. PLoS Med. 2008, 5:e133.

6. Rosenthal, P. J. Curr. Opin. Hematol. 2002, 9, 140.

7. Mauritz, J. M.; Seear, R.; Esposito, A.; Kaminski, C. F.; Skepper, J. N.; Warley, A.; Lew V. L.; Tiffert, T. Biophys. J. 2011, 100, 1438.

8. Naughton, J. A.; Nasizadeh, S.; Bell, A. Mol. Biochem. Parasitol. 2010, 173, 81.

9. Krugliak, M.; Zhang, J.; Ginsburg, H. Mol. Biochem. Parasitol. 2002, 119, 249.

10. Payne, S. H.; Loomis, W. F. Eukaryot. Cell. 2006, 5, 272.

11. Liu, J.; Istvan, E. S.; Gluzman, I. Y.; Gross, J.; Goldberg, D. E. Proc. Natl. Acad. Sci. U S A, 2006, 103, 8840 .

12. Martin, R. E.; Kirk, K. Blood. 2007, 109, 2217.

13. Goldberg, D. E. Curr. Top. Microbiol. Immunol. 2005, 295, 275.

14. Wunderlich, J.; Rohrbach, P.; Dalton, J. P. Frontiers of Bioscience. (in press).

15. Rohrbach, R. Biotechnol. J. 2009, 4, 812.

16. Kuhn, Y.; Rohrbach, P.; M. Lanzer, M. Cell. Microbiol. 2007, 9, 1004.

17. Rosenthal, P. J. Int. J. Parasitol. 2004, 34, 1489.

18. Rosenthal, P. J. Adv. Exp. Med. Biol. 2011, 712, 30.

19. Liu, J.; Gluzman, I. Y.; Drew, M. E.; Goldberg, D. E. J. Biol. Chem. 2005, 280, 1432. 
20. Kolakovich, K. A.; Gluzman, I. Y.; Duffin, K. L.; Goldberg, D. E. Mol. Biochem. Parasitol. 1997, 87, 123.

21. Ersmark, K.; Samuelsson, B.; Hallberg, A. Med. Res. Rev. 2006, 26, 626.

22. Omara-Opyene, A. L.; Moura, P. A.; Sulsona, C. R.; Bonilla, J. A.; Yowell, C. A.; Fujioka, H.; Fidock, D. A.; Dame, J. B. J. Biol. Chem. 2004, 279, 54088.

23. Sijwali, P. S.; Rosenthal, P. J. Proc. Natl. Acad. Sci. U S A. 2004, 101, 4384.

24. Ersmark, K.; Feierberg, I.; Bjelic, S.; Hamelink, E.; Hackett, F.; Blackman, M. J.; Hultén, J.; Samuelsson, B.; Aqvist, J.; Hallberg, A. J. Med. Chem. 2004, 47, 110.

25. Klemba, M.; Gluzman, I.; Goldberg, D. E. J. Biol. Chem. 2004, 279, 43000.

26. Wang, F.; Krai, P.; Deu, E.; Bibb, B.; Lauritzen, C.; Pedersen, J.; Bogyo, M.; Klemba, M. Mol. Biochem. Parasitol. 2011, 175, 10.

27. Curley, G. P.; O'Donovan, S. M.; McNally, J.; Mullally, M.; O'Hara, H.; Troy, A.; O'Callaghan, S. A.; Dalton, J. P. J. Eukaryot. Microbiol. 1994, 41, 119.

28. Nankya-Kitaka, M. F.; Curley, G. P.; Gavigan, C. S.; Bell, A.; Dalton, J. P. Parasitol. Res. 1998, 84, 552.

29. Gavigan, C. S.; Dalton, J. P.; Bell, A. Mol Biochem Parasitol 2001, 117, 37.

30. Dalal, S.; Klemba, M. J. Biol. Chem. 2007, 282, 35978.

31. Skinner-Adams, T. S.; Stack, C. M.; Trenholme, K. R.; Brown, C. L.; Grembecka, J.; Lowther, J.; Mucha, A.; Drag, M.; Kafarski, P.; McGowan, S.; Whisstock, J. C.; Gardiner, D. L.; Dalton, J. P. Trends Biochem. Sci. 2009, 35, 53.

32. Raghed, D.; Bompiani, K.; Dalal, S.; Klemba, M. J. Biol. Chem. 2009, 284, 24806.

33. Ragheb, D.; Dalal, S.; Bompiani, K. M.; Ray, W. K.; Klemba, M. J. Biol. Chem. 2011, 286, 27255.

34. Gardiner, D. L.; Skinner-Adams, T. S.; Brown, C. L.; Andrews, K. T.; Stack, C. M.; McCarthy, J. S.; Dalton, J. P.; Trenholme, K. R. Expert Rev. Anti. Infect. Ther. 2009, 7,1087.

35. Charet, P.; Aissi, E.; Maurois, P.; Bouquelet, S.; Biguet, J. Comp. Biochem. Physiol. 1980, $65 B, 519$.

36. Gyang, F. N.; Poole, B.; Trager, W. Mol. Biochem. Parasitol. 1982, 5, 263.

37. Slominanny, C.; Charet, P.; Prensier, G. J. Protozool. 1983, 30, 376.

38. Vander Jagt, D. L.; Baack, B. R.; Hunsaker, L. A. Mol. Biochem. Parasitol. 1984, 10, 45.

39. Vander Jagt, D. L.; Hunsaker, L. A.; Campos, N. M. Biochem. Pharmacol. 1987, 36, 3285.

40. Florent, I.; Derhy, Z.; Allary, M.; Monsigny, M.; Mayer, R.; Schrével, J. Mol. Biochem. Parasitol. 1998, 97, 149.

41. Stack, C. M.; Lowther, J.; Cunningham, E.; Donnelly, S.; Gardiner, D. L.; Trenholme, K. R.; Skinner-Adams, T. S.; Teuscher, F.; Grembecka, J.; Mucha, A.; Kafarski, P.; Lua, L.; Bell, A.; Dalton, J. P. J. Biol. Chem. 2007, 282, 2069.

42. McGowan, S.; Porter, C. J.; Lowther, J.; Stack, C. M.; Golding, S. J.; Skinner-Adams, T. S.; Trenholme, K. R.; Teuscher, F.; Donnelly, S. M.; Grembecka, J.; Mucha, A.; Kafarski, P.; Degori, R.; Buckle, A. M.; Gardiner, D. L.; Whisstock, J. C.; Dalton, J. P. Proc. Natl. Acad. Sci. U S A. 2009, 106, 2537. 
43. McGowan, S.; Oellig C. A.; Birru1, W.A.; Caradoc-Davies, T. T.; Stack C. M.; Lowther, J.; Skinner-Adams, T.; Mucha, A.; Kafarski, P.; Grembecka, J.; Trenholme, K. R.; Buckle, A. M.; Gardiner, D. L.; Dalton, J. P.; Whisstock, J. C. Proc. Natl. Acad. Sci. U S A. 2010, 107, 2449.

44. Velmourougane, G.; Harbut, M. B.; Dalal, S.; McGowan, S.; Oellig, C. A.; Meinhardt, N.; Whisstock, J. C.; Klemba, M.; Greenbaum, D. J. Med. Chem. 2011, 54, 1655.

45. Harbut, M. B.; Velmourougane, G.; Dalal, S.; Reiss, G.; Whisstock, J. C.; Onder, O.; Brisson, D.; McGowan, S.; Klemba, M.; Greenbaum, D. Proc. Natl. Acad. Sci. U S A. 2011, $108,526$.

46. Maric, S.; Donnelly, S. M.; Robinson, M. W.; Skinner-Adams, T.; Trenholme, K. R.; Gardiner, D. L.; Dalton, J. P.; Stack, C. M.; Lowther, J. Biochemistry. 2009, 48, 5435.

47. Mucha, A.; Drag, M.; Dalton, J. P.; Kafarski, P. Biochimie. 2010, 92, 1509.

48. Grembecka, J.; Mucha, A.; Cierpicki, T.; Kafarski, P. J. Med. Chem. 2003, 46, 2641.

49. Skinner-Adams, T. S.; Lowther, J.; Teuscher, F.; Stack, C. M.; Grembecka, J.; Mucha, A.; Kafarski, P.; Trenholme, K. R.; Dalton, J. P.; Gardiner, D. L. J. Med. Chem. 2007, 50, 6024.

50. Flipo, M.; Beghyn, T.; Leroux, V.; Florent, I.; Deprez, B. P.; Deprez-Poulain, R. F. J. Med. Chem. 2007, 50, 1322.

51. Cunningham, E.; Drag, M.; Kafarski, P.; Bell, A. Antimicrob. Agents Chemother. 2008, 52, 3221.

52. Gardiner, D. L.; Trenholme, K. R.; Skinner-Adams, T. S.; Stack, C. M.; Dalton, J. P. J. Biol. Chem. 2006, 281, 1741.

53. Allary, M.; Schrevel, J.; Florent, I. Parasitology. 2002, 125, 1.

54. Azimzadeh, O.; Sow, C.; Gèze, M.; Nyalwidhe, J.; Florent, I. Malar J. 2010, 9, 189.

55. Teuscher, F.; Lowther, J.; Skinner-Adams, T.; Spielmann, T., Dixon, M. W.; Stack, C. M.; Donnelly, S.; Mucha. A.; Kafarski, P.; Vassiliou, S.; Gardiner, D. L.; Dalton, J.P.; Trenholme, K. R. J. Biol. Chem. 2007, 282, 30817.

56. Lamarque, M.; Tastet, C.; Poncet, J.; Demettre, E.; Jouin, P.; Vial, H.; Dubremetz, J. F. Proteomics Clin. Appl. 2008, 2, 1361.

57. Jones, P. M.; Robinson, M. W.; Dalton, J. P.; George, A. M. PLoS One (in press).

58. Taylor, A. FASEB J., 1993, 7, 290.

59. Fidock, D. A.; Eastman, R. T.; Ward, S. A.; Meshnick, S. R. Trends Parasitol. 2010, 24, 537.

60. RTS, S Clinical Trials Partnership. N. Engl. J. Med. 2011, 365,1863.

61. Noedl, H.; Socheat, D.; Satimai, W. N. Eng. J. Med. 2009, 361, 540.

62. Dondorp, A. M.; Nosten, F.; Yi, P.; Das, D.; Phyo, A. P.; Tarning, J.; Lwin, K. M.; Ariey, F.; Hanpithakpong, W.; Lee, S. J.; Ringwald, P.; Silamut, K.; Imwong, M.; Chotivanich, K.; Lim, P.; Herdman, T.; An, S. S.; Yeung, S.; Singhasivanon, P.; Day, N. P. J.; Lindegardh, N.; Socheat, D.; White, N. J. N. Eng. J. Med. 2009, 361, 455.

63. Gavigan, C. S.; Machado, S. G.; Dalton, J. P.; Bell, A. Antimicrob. Agents Chemother. 2001, 45,3175 . 
64. Krige, D.; Needham, L. A.; Bawden, L. J.; Flores, N.; Farmer, H.; Miles, L. E.; Stone, E.; Callaghan, J.; Chandler, S.; Clark, V. L.; Kirwin-Jones, P.; Legris, V.; Owen, J.; Patel, T.; Wood, S.; Box, G.; Laber, D.; Odedra, R.; Wright, A.; Wood, L. M.; Eccles, S. A.; Bone, E. A.; Ayscough, A.; Drummond, A. H. Cancer Res. 2008, 68, 6669.

65. Löwenberg, B.; Morgan, G.; Ossenkoppele, G. J.; Burnett, A. K.; Zachée, P.; Dührsen, U.; Dierickx, D.; Müller-Tidow, C.; Sonneveld, P.; Krug, U.; Bone, E.; Flores, N; Richardson, A. F.; Hooftman, L.; Jenkins, C.; Zweegman, S.; Davies, F. J. Clin. Oncol. 2010, 28, 4333.

66. Ichinose, Y.; Genka, K.; Koike, T.; Kato, H.; Watanabe, Y.; Mori, T.; Iioka, S.; Sakuma, A.; Ohta, M. J. Nat. Cancer Instit. 2003, 95, 605. 